\title{
Identification of factors affecting the surgical margin in wedge resection using preoperative lipiodol marking
}

\author{
Satoshi Fumimoto, Kiyoshi Sato, Nobuharu Hanaoka, Takahiro Katsumata \\ Department of Thoracic and Cardiovascular Surgery, Osaka Medical College, Osaka, Japan \\ Contributions: (I) Conception and design: S Fumimoto; (II) Administrative support: S Fumimoto; (III) Provision of study materials or patients: N \\ Hanaoka; (IV) Collection and assembly of data: S Fumimoto; (V) Data analysis and interpretation: S Fumimoto; (VI) Manuscript writing: All authors; \\ (VII) Final approval of manuscript: All authors. \\ Correspondence to: Satoshi Fumimoto, MD, PhD. Department of Thoracic and Cardiovascular Surgery, Osaka Medical College, 2-7 Daigaku-cho, \\ Takatsuki, Osaka 569-8686, Japan. Email: tho089@osaka-med.ac.jp.
}

\begin{abstract}
Background: The factors affecting the surgical margin distance in resection of small lung lesions after preoperative marking are still unclear. The purpose of this study was to identify these factors in wedge lung resection using a localization technique.

Methods: The subjects were 45 patients with small pulmonary nodules who underwent preoperative computed tomography-guided lipiodol marking followed by video-assisted thoracoscopic surgery between April 2017 and December 2019. Data were obtained for nodule size, depth from the pleural surface, imaging features, and procedure-related factors that could affect the surgical margin. Subjects were divided into groups with margin distances $<10$ and $\geq 10 \mathrm{~mm}$. Logistic regression analysis was used to identify factors associated with the margin distance.

Results: Preoperative marking was performed using lipiodol prior to resection of 52 nodules (median size, $10.1 \mathrm{~mm}$; range, $6.75-12.3 \mathrm{~mm}$ ) in 45 patients ( $23 \mathrm{men}, 22$ women; median age, 65.4 years). The mean distance from the pleural surface to the pulmonary lesion was $13.8 \mathrm{~mm}$ (range, 5.44-22.2 $\mathrm{mm}$ ). The 3D deviation of the radio-opaque nodule (lipiodol spot) from the lesion was the only significant difference in nodule- and procedure-related factors between the two groups. Multivariate analysis also showed that this $3 \mathrm{D}$ deviation was the most significant factor affecting the margin distance (odds ratio, 0.26; $95 \%$ CI, 0.08$0.81 ; \mathrm{P}=0.02)$.

Conclusions: The findings in this study may help to ensure a sufficient surgical margin after preoperative lipiodol marking, through recognition that the $3 \mathrm{D}$ deviation of the radio-opaque nodule from the target lesion has a particularly important influence on the margin distance.
\end{abstract}

Keywords: Small pulmonary nodule; surgical margin distance; wedge resection; preoperative marking

Submitted Feb 01, 2021. Accepted for publication Apr 09, 2021.

doi: $10.21037 /$ jtd-21-211

View this article at: http://dx.doi.org/10.21037/jtd-21-211

\section{Introduction}

The increased use of computed tomography (CT)-based screening for lung cancer has resulted in more frequent detection of small pulmonary nodules (SPNs) $(1,2)$. Diagnosis and treatment of SPNs have become important issues because these nodules have malignant potential and heterogeneous characteristics (3). Video-assisted thoracoscopic surgery (VATS) is a safe and minimally invasive procedure for diagnostic biopsy and therapeutic resection (4). However, localization of SPNs for VATS is often challenging because of the inability to detect the lesions visually or palpate them directly $(5,6)$. Thus, many methods have been developed for preoperative localization of SPNs, such as those using a dye (7), barium (8), lipiodol (9-15), a microcoil (16), and a hook wire (17). An optimal 
localization method has yet to be established, but most of these techniques have been reported to be accurate and safe, and are associated with low complication rates and high success rates (18).

For evaluation of the surgical margin, most studies define the safety margin of wedge resection as the negative margin in preoperative localization. However, few studies have evaluated the distance from the tumor nodule to the staple line. In wedge resection, the margin distance is associated with a risk of local recurrence (19-24). Even in a case with a negative resection margin, an insufficient resection margin may influence local recurrence and survival (19). Furthermore, depending on the distance from the pleura and localization of the target lesion, it may not be possible to secure a sufficient surgical margin (19). Several studies have shown that the risk of local recurrence decreases as the margin distance increases (19-24), but to our knowledge, the surgical margin distance in wedge resection following preoperative marking has not been examined.

We have used a combined approach of preoperative lipiodol marking and surgical resection for SPNs in a hybrid operating room since June 2016 at Osaka Medical College Hospital (25). In this study, we retrospectively examined the surgical margin distance of resected lung parenchyma after preoperative localization and VATS resection. The aim of the study was to evaluate this distance and identify factors that affect the surgical margin in preoperative lipiodol marking.

We present the following article in accordance with the STROBE reporting checklist (available at http://dx.doi. org/10.21037/jtd-21-211).

\section{Methods}

\section{Patients and eligibility for the study}

The study was conducted in accordance with the Declaration of Helsinki (as revised in 2013). This retrospective study was approved by the institutional review board of Osaka Medical College Hospital and informed consent was obtained from each patient (approval No. 1905). From April 2017 to December 2019, a total of 45 patients (52 nodules) underwent combined lipiodol marking and VATS resection in a hybrid operating room. The indication for pulmonary localization was determined by thoracic surgeons. Nodules that were considered difficult to localize during thoracoscopy, such as GGO lesions, nodules located at a considerable depth from the pleural surface, and nodules less than $10 \mathrm{~mm}$ in size, were candidates for this technique. The following nodules were excluded: (I) nodules located within the inner two third of the lung; (II) nodules located within $4 \mathrm{~mm}$ from the pleural surface to edge of nodule. Because our interest was in the association between the surgical margin distance and preoperative marking, we restricted the analysis in this study to patients who underwent wedge resection.

\section{Marking technique}

All procedures were performed in a hybrid operating room equipped with a sliding gantry-based CT system (Somatom Definition AS+SLID, Siemens, Germany). One surgical team, including a board-certified thoracic surgeon (S.F.) with experience in this method, performed both marking and surgery. The procedural workflow has been described elsewhere (25). After induction of general anesthesia, the patient was placed in a supine or prone position to establish an optimal access route for needle insertion, depending on the location of the SPN. Scale paper with a metal wire was firmly placed on the patient and a slide gantry-based CT scan was performed. The shortest distance from the nodule to the chest wall was chosen as the injection site. A 23-gauge needle was introduced through the skin into the perilesional SPN while avoiding structures such as blood vessels and bronchi, as well as tumors. The syringe was withdrawn to ensure that no blood or air had refluxed, respiratory ventilation was stopped, and $0.2 \mathrm{~mL}$ of lipiodol (Lipiodol UltraFluid; Laboratory Guerbet; Aulnay-SousBois, France) was carefully injected in a single shot adjacent to the lesion. Immediately after injection of lipiodol, wholechest CT images were obtained to evaluate the lipiodol spot (Figure 1A). Thereafter, reconstruction of the location of the SPN and radio-opaque nodule was performed using the 3D volume-rendering method (Synapse-Vincent, Fujifilm Medical Co., Tokyo, Japan). The accuracy of lipiodol marking was assessed by measuring the deviation from the center of the lipiodol spot to the center of the target SPN after 3D image reconstruction (Figure 1B).

\section{Surgical treatment}

Immediately after localization, the patient was repositioned into the lateral decubitus position. VATS via 2 or 3 ports was performed with one-lung ventilation. Thereafter, a C-arm-shaped fluoroscopy unit was placed horizontally in an appropriate position. Finger palpation to identify 

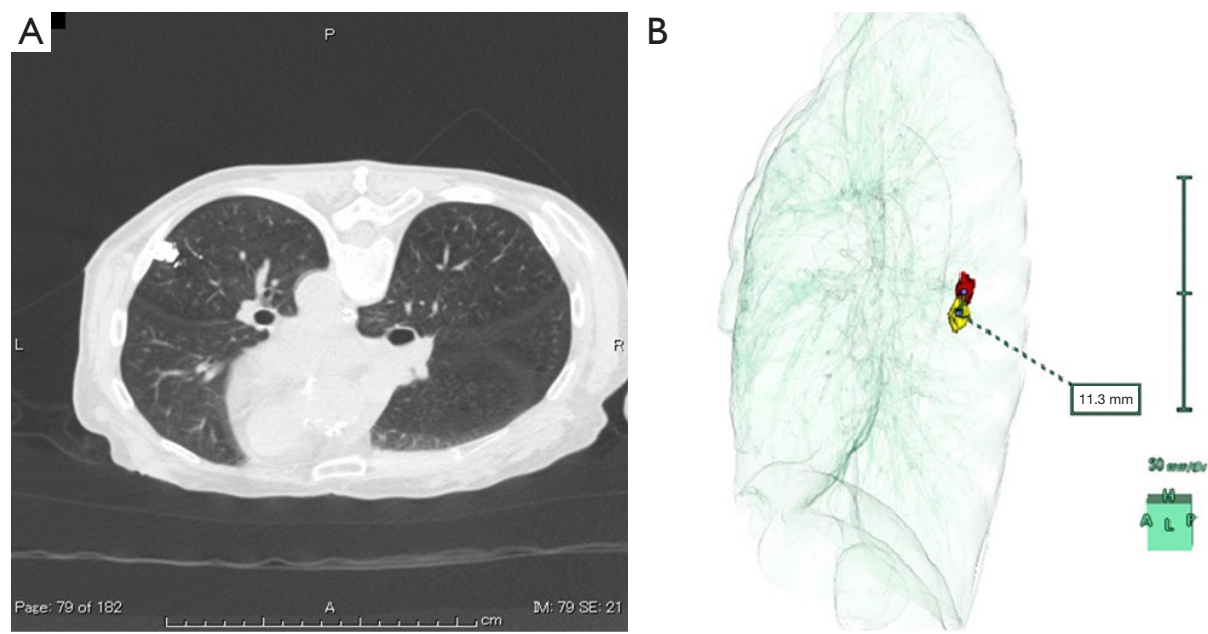

Figure 1 Computed tomography (CT) image and reconstructed 3D image taken after lipiodol marking. (A) A volume of $0.2 \mathrm{~mL}$ of lipiodol was injected adjacent to the target lesion. The lipiodol spot was confirmed on CT. (B) The target lesion (red) and radio-opaque nodule (yellow) with accumulated lipiodol. The deviation was measured as the distance between the centers of the target lesion and the nodule on $3 \mathrm{D}$ CT.

SPNs was not performed in any cases. The lipiodol spot was grasped with ring-shaped forceps during fluoroscopy and then resected with an endoscopic stapler. Successful resection was confirmed by viewing the lipiodol spot within the resected specimen under C-arm fluoroscopy. After VATS wedge resection, frozen section analysis of the resected lesion was performed.

\section{Endpoints}

The primary endpoint was achievement of complete resection, which was defined as the absence of tumor cells at the resected margin, excluding the staple line. The secondary endpoint was defined as acquisition of a sufficient surgical margin distance, which was in turn defined as the distance from the tumor to the nearest resection line on a totally deflated lung with staples removed. The cut staple line removes $0.5 \mathrm{~cm}$. The distance was measured macroscopically by a pathologist and recorded on the final pathology report. Patients were divided into two groups based on a surgical margin distance of $<10$ and $\geq 10 \mathrm{~mm}$.

\section{Statistical analysis}

Normally distributed continuous variables are presented as means [95\% confidence interval (CI)] and compared using a 2 -sample (unpaired) Student t test. Skewed variables are summarized as medians [interquartile range (IQR)] and compared by Mann-Whitney $U$ test. Univariate and multivariate logistic regression analyses were performed to determine the association of nodule-related and procedurerelated characteristics with the secondary endpoint. In univariate analysis, the baseline characteristics included the $3 \mathrm{D}$ deviation of the lipiodol spot from the lesion, CT findings, lesion location, depth-to-size ratio, patient position for marking, and surgical procedures. $\mathrm{P}<0.05$ was considered to be statistically significant. All analyses were performed with SPSS (v.20.0; IBM SPSS Inc., Armonk, NY, USA).

\section{Results}

The general characteristics of the 45 subjects are shown in Table 1. Most of the patients $(39 / 45,86.7 \%)$ had one nodule, $5(11.1 \%)$ had two nodules, and $1(2.2 \%)$ had three nodules. The characteristics of the target nodules are shown in Table 2. Based on preoperative CT, 16 SPNs (31\%) were classified as solid lesions, and all others were categorized as GGO or part-solid GGO lesions. The median size of the SPNs on preoperative CT images was $10.1 \mathrm{~mm}$ (range, $6.75-12.3 \mathrm{~mm}$ ); the mean distance from the pleural surface was $13.8 \mathrm{~mm}$ (range, 5.44-22.2 $\mathrm{mm}$ ), and the median tumor depth-to-size ratio was $1.84(0.67-2.36)$. These variables did not differ significantly between the groups with margin distances of $<10$ and $\geq 10 \mathrm{~mm}$.

Details of the marking and surgical procedure are shown in Table 3. Lipiodol accumulation was detectable on CT and intraoperative fluoroscopy for all target nodules. VATS was 
performed without conversion to thoracotomy in all cases. All specimens had an initially negative surgical margin, and the mean margin distance was $13.3 \mathrm{~mm}$ (range, 7.27-19.3 mm). The $3 \mathrm{D}$ deviation from the center of the lipiodol spot to

Table 1 Patient characteristics

\begin{tabular}{lc}
\hline Variables & Number \\
\hline Total patient number, $\mathrm{n}$ & 45 \\
Sex, $\mathrm{n}(\%)$ & $23(51.1)$ \\
Male & $22(48.9)$ \\
Female & $65.4(56.3-75.3)$ \\
Age (years) (IQR) & $22.8(20.7-24.2)$ \\
Body mass index (IQR) & $74.3(68.6-78.8)$ \\
FEV $1.0 \%$ (IQR) & \\
Number of lesions, $\mathrm{n}(\%)$ & $39(86.7)$ \\
One & $5(11.1)$ \\
Two & $1(2.2)$ \\
Three & \\
\hline
\end{tabular}

Data are given as counts (percentages in parentheses) or mean (standard deviations in parentheses). FEV1.0\%, percentage of forced expiratory volume in 1 second; IQR, interquartile ratio. the center of the target SPN differed significantly in the two groups (margin $<10 \mathrm{~mm}$ : $16.2 \mathrm{~mm} v$ s. margin $\geq 10 \mathrm{~mm}$ : $12.2 \mathrm{~mm}, \mathrm{P}=0.03)$. The distance to the pleural surface and tumor size was larger for those lesions with margin $<10 \mathrm{~mm}$ although neither factor individually was statistically significant in this study. Other variables, including time required for marking, size of lipiodol spot, and operation time, did not show a significant difference between the groups.

The $3 \mathrm{D}$ deviation was the only factor that was significantly associated with the margin distance in univariate (OR, 0.35; 95\% CI, 0.13-0.96; $\mathrm{P}=0.04)$ and multivariate $(\mathrm{OR}, 0.26$; 95\% CI, 0.08-0.81; $\mathrm{P}=0.02)$ analyses (Table 4).

\section{Discussion}

In this study, we investigated factors that may influence the surgical margin distance in preoperative lipiodol marking. Both technical factors related to marking and the surgical procedure and tumor factors such as location, size, and depth were evaluated. However, in statistical analysis, only the $3 \mathrm{D}$ deviation between the lipiodol spot and the lesion was a significant factor affecting the margin distance.

Table 2 Characteristics of the nodules

\begin{tabular}{|c|c|c|c|c|}
\hline Variable & Entire cohort & Margin $\geq 10 \mathrm{~mm}$ & Margin $<10 \mathrm{~mm}$ & $P$ value \\
\hline CT findings & & & & NA \\
\hline GGO & 28 & 20 & 8 & \\
\hline Part-solid GGO & 8 & 6 & 2 & \\
\hline Lesion location & & & & NA \\
\hline RUL & 13 & 10 & 3 & \\
\hline RML & 4 & 3 & 1 & \\
\hline RLL & 15 & 9 & 6 & \\
\hline Lesion size on $\mathrm{CT}$, mm (IQR) & $10.1(6.75-12.3)$ & $9.0(7.0-11.3)$ & $7.5(5.8-16.3)$ & 0.58 \\
\hline Distance to the pleural surface, $\mathrm{mm}(95 \% \mathrm{Cl})$ & $13.8(5.44-22.2)$ & $13.5(4.92-22.1)$ & $14.5(6.46-22)$ & 0.67 \\
\hline
\end{tabular}

Data are given as counts (percentages in parentheses) or mean (standard deviations in parentheses). NA: not available; GGO: ground glass opacity; RUL: right upper lobe; RML: right middle lobe; RLL: right lower lobe; LUL: left upper lobe; LLL: left lower lobe; IQR: interquartile ratio; Cl: confidence interval. 
Table 3 Procedure and pathological details

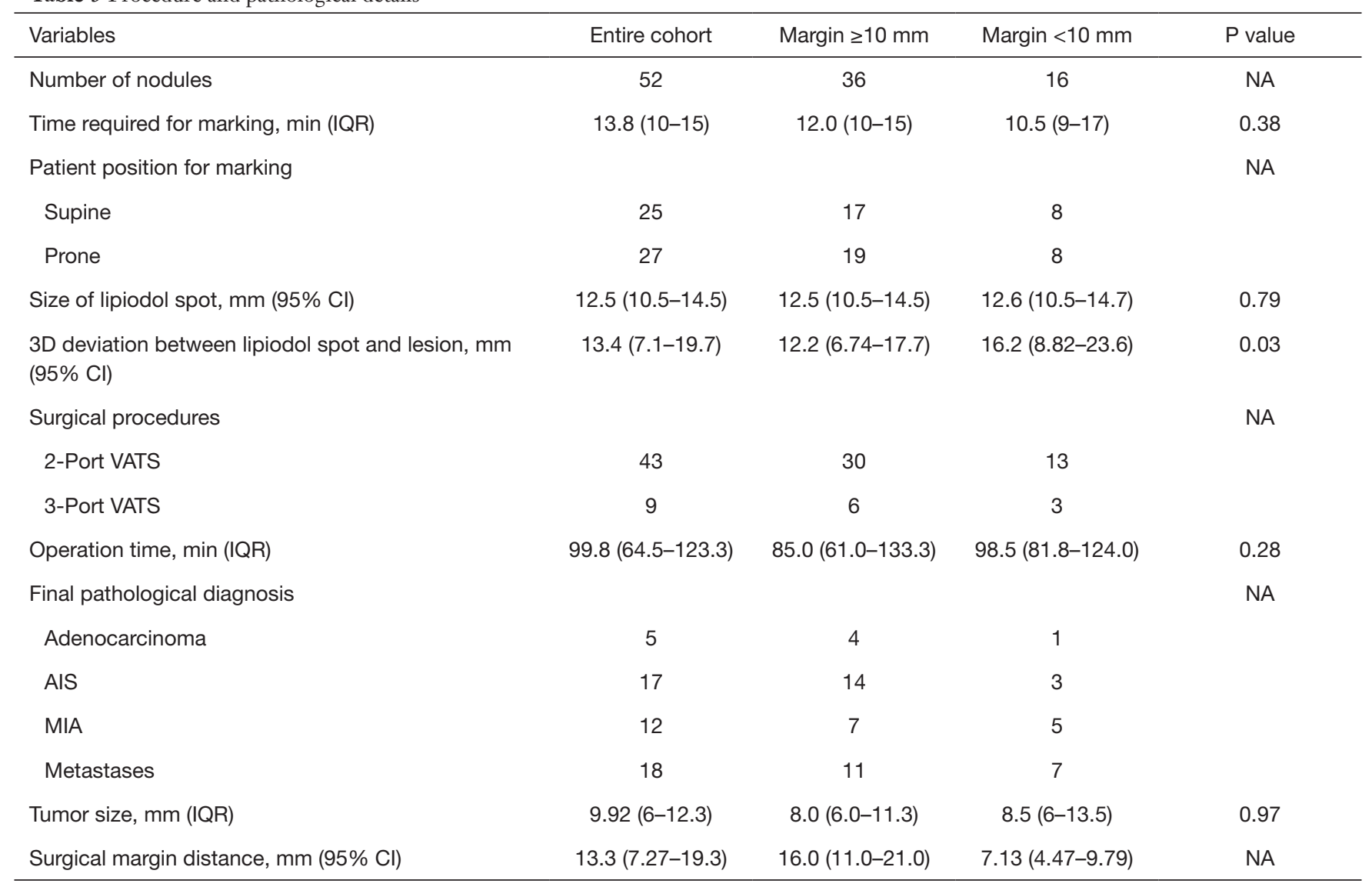

Data are given as counts (percentages in parentheses) or mean (standard deviations in parentheses). NA, not available; VATS, videoassisted thoracoscopic surgery; AIS, adenocarcinoma in situ; MIA, minimum invasive adenocarcinoma; IQR, interquartile ratio; CI, confidence interval.

Limited resection, including wedge resection and segmentectomy, has typically been the choice for patients with poor cardiopulmonary function (19). With recent advances in CT, SPNs invisible on chest radiography are increasingly being detected. Despite a lack of sufficient evidence, intentional limited resection is increasingly indicated for low-risk patients with early-stage lung cancer (24). For limited resection, evaluation of the surgical margin distance is a major concern in terms of local control. An inadequate margin increases local recurrence, with the potential for microscopic spread of cancer cells from the margin (19-24). Thus, El-Sherif et al. reported a higher rate of local recurrence in cases in which the margin was $<10 \mathrm{~mm}$ compared with $\geq 10 \mathrm{~mm}$ (20); and Mohiuddin et al. found that the local recurrence risk among patients with a $10 \mathrm{~mm}$ margin was $45 \%$ lower than that in patients with a $5 \mathrm{~mm}$ margin (21). Wolf et al. also reported that the margin distance was independently associated with longer survival and recurrence-free survival, as well as local recurrence (22).

The findings in the studies above are mainly based on resection of early-stage lung cancer, but similar results have been reported for pulmonary metastasis (23). Thus, since a margin of $<10 \mathrm{~mm}$ has been significantly associated with the local recurrence rate, ensuring margins $\geq 10 \mathrm{~mm}$ should be a priority in limited resection to achieve local control $(20-22,24)$. For this reason, we divided our patients into two subgroups with margins of $<10$ and $\geq 10 \mathrm{~mm}$, based on pathological reports of the surgical margin distance.

Preoperative marking is useful to localize SPNs and permits wedge resection by VATS. However, during preoperative marking, infrequent positive resection margins and recurrence at the surgical stump have been reported (7). A systematic review and meta-analysis compared the three most widely used preoperative 
Table 4 Univariate and multivariate analyses of factors affecting the distance of the surgical margin

\begin{tabular}{|c|c|c|c|c|}
\hline Variables & Univariate OR $(95 \% \mathrm{Cl})$ & $P$ value & Multivariate OR $(95 \% \mathrm{Cl})$ & $P$ value \\
\hline \multicolumn{5}{|l|}{ CT findings } \\
\hline GGO/part-solid lesion vs. solid lesion & $1.56(0.45-5.43)$ & 0.49 & $0.83(0.16-4.36)$ & 0.82 \\
\hline \multicolumn{5}{|l|}{ Lesion location } \\
\hline \multicolumn{5}{|l|}{ Patient position for marking } \\
\hline Supine vs. prone & $1.61(0.49-5.26)$ & 0.43 & $0.29(0.06-1.39)$ & 0.12 \\
\hline \multicolumn{5}{|l|}{ Surgical procedure } \\
\hline 2-port vs. 3-port & $0.87(0.19-4.01)$ & 0.86 & $0.84(0.13-5.32)$ & 0.85 \\
\hline
\end{tabular}

marking techniques (hook wire, microcoil and lipiodol), but did not provide a detailed assessment of the surgical margin (18). Many studies have evaluated factors associated with success rates and complication rates in preoperative localization, but the current study is novel in its focus on the surgical margin distance. The first nonrandomized prospective study comparing two marking methods concluded that use of a hook wire resulted in more initially positive resection margins than use of lipiodol due to the difficulty in determining the exact location of a lesion in the lung parenchyma (17). With lipiodol, such a lesion can be resected under fluoroscopy and confirmation that the lesion is included in the specimen is possible through the C-arm after resection (9-15).

In the current study, tumor factors such as size, distance from the pleural surface, and components did not affect the surgical margin distance. Unlike other localization techniques that only label the pleural surface, lipiodol accurately indicates the resection area under real-time fluoroscopy guidance, especially for deep-seated lesions $(17,26)$. This property of lipiodol may explain why the lesion characteristics did not affect the surgical margin distance. The margin was also unaffected by the position at the time of marking and the number of ports in VATS, and the $3 \mathrm{D}$ deviation was the only factor affecting the margin. The distance between the needle tip and the nodule on chest CT has been measured for assessment of marking accuracy (26), but considering the 3D shape of the lung, a distance measured in either the axial or coronal view may not reflect the exact distance from the radio-opaque nodule to the lesion. In this study, we reconstructed 3D images from chest $\mathrm{CT}$ and measured the $3 \mathrm{D}$ deviation to understand the precise positional relationship between the lesion and radio-opaque nodule. Univariate and multivariate analyses showed that the $3 \mathrm{D}$ deviation of the radio-opaque nodule from the pulmonary lesion was the most significant factor affecting the surgical margin distance.

In percutaneous preoperative marking, direct puncture of lung lesions may cause pleural dissemination and should be avoided (14). Lipiodol itself poses a potential risk of systemic embolism because it is a water-insoluble material $(10,15)$. Lipiodol marking also has a potential risk of air migration into adjacent vessels. Therefore, it is safer to inject lipiodol into the lung parenchyma, where there are few structures such as thick blood vessels and bronchi, to prevent embolism $(9-15,25,26)$. However, the deeper the tumor is located from the pleural surface, the closer it is to the hilum and the thicker the blood vessels and bronchi. Thus, to puncture a lung parenchyma safely, lipiodol must be injected away from the lesion. This study suggests that if the $3 \mathrm{D}$ deviation is low, a sufficient surgical margin can be secured, but that a greater deviation may result in an insufficient surgical margin. Because the radiopaque shadow generally created by lipiodol is vague, the distance between the lesion and the radiopaque nodule may affect the postoperative margin. This implies that inaccurate lipiodol marking may lead to smaller surgical margin. As we accumulate more cases in the near future, we may be able to clarify the factors that affect the distance of $3 \mathrm{D}$ deviation. 


\section{Limitations}

The study has some limitations. First, it was performed as a single-center, retrospective study, so selection bias could not be avoided, and the relatively small number of patients weakened the statistical data. Second, unlike conventional preoperative marking, our localization was performed under general anesthesia in a hybrid operating room. This approach may have a lower incidence of complications such as pneumothorax and hemoptysis compared to the traditional approach (25,27-29), and may not affect the marking technique itself, but further direct comparison of the two approaches is still needed. However, a singlecenter study found no differences in marking success rates or complication rates (29), with the conclusion that the choice between the two approaches should be based on the most readily available approach at each facility, since no significant difference in outcomes was found. Therefore, we believe that our marking approach did not affect the results. Third, measuring a precise margin distance is frequently difficult due to the presence of staples, and there is no standard method to assess the surgical margin (30). We measured from the cut staple line, which may add approximately $0.5 \mathrm{~cm}$ to measurements taken before the cut, on a deflated lung. Thus, our measured distance may be shorter than that found in other studies of the surgical margin.

\section{Conclusions}

The $3 \mathrm{D}$ deviation between the radio-opaque nodule and the target lesion affected the surgical margin distance after preoperative lipiodol marking, but other tumor and technical factors had no significant effects. These results suggest that decreasing the $3 \mathrm{D}$ deviation will produce a more accurate surgical margin, regardless of the tumor size and depth, and technical factors. Also, measurement of the $3 \mathrm{D}$ deviation between the lipiodol nodule and the target nodule may be a useful indicator for the safety and accuracy of resection of SPNs.

\section{Acknowledgments}

The data in the study were presented at the 37th Annual Meeting of the Japanese Association for Chest Surgery, Tokyo, Japan, September 2020.

Funding: None.

\section{Footnote}

Reporting Checklist: The authors have completed the STROBE reporting Checklist. Available at http://dx.doi. org/10.21037/jtd-21-211

Data Sharing Statement: Available at http://dx.doi. org/10.21037/jtd-21-211

Peer Review File: Available at http://dx.doi.org/10.21037/jtd21-211

Conflicts of Interest: All authors have completed the ICMJE uniform disclosure form (available at http://dx.doi. org/10.21037/jtd-21-211). The authors have no conflicts of interest to declare.

Ethical Statement: The authors are accountable for all aspects of the work in ensuring that questions related to the accuracy or integrity of any part of the work are appropriately investigated and resolved. The study was approved by the institutional review board of Osaka Medical College Hospital, No. 1905. The requirement for written consent was waived because of the retrospective nature of the study. The study was conducted in accordance with the Declaration of Helsinki (as revised in 2013).

Open Access Statement: This is an Open Access article distributed in accordance with the Creative Commons Attribution-NonCommercial-NoDerivs 4.0 International License (CC BY-NC-ND 4.0), which permits the noncommercial replication and distribution of the article with the strict proviso that no changes or edits are made and the original work is properly cited (including links to both the formal publication through the relevant DOI and the license). See: https://creativecommons.org/licenses/by-nc-nd/4.0/.

\section{References}

1. MacMahon H, Naidich DP, Goo JM, et al. Guidelines for management of incidental pulmonary nodules detected on CT images: from the Fleischner Society 2017. Radiology 2017;284:228-43.

2. Detterbeck FC, Mazzone PJ, Naidich DP, et al. Screening for lung cancer: Diagnosis and management of lung cancer, 3 rd ed: American College of Chest Physicians evidence-based clinical practice guidelines. 
Chest 2013;143:e78S-92S.

3. Pedersen JH, Saghir Z, Wille MM, et al. Groundglass opacity lung nodules in the era of lung cancer CT screening: radiology, pathology, and clinical management. Oncology (Williston Park) 2016;30:266-74.

4. Congregado M, Merchan RJ, Gallardo G, et al. Videoassisted thoracic surgery (VATS) lobectomy: 13 years' experience. Surg Endosc 2008;22:1852-7.

5. Nakashima S, Watanabe A, Obama T, et al. Need for preoperative computed tomography-guided localization in video-assisted thoracoscopic surgery pulmonary resections of metastatic pulmonary nodules. Ann Thorac Surg 2010;89:212-8.

6. Suzuki K, Nagai K, Yoshida J, et al. Video-assisted thoracoscopic surgery for small indeterminate pulmonary nodules: indications for preoperative marking. Chest 1999;115:563-8.

7. Sato M, Omasa M, Chen F, et al. Use of virtual assisted lung mapping (VAL-MAP), a bronchoscopic multispot dye-marking technique using virtual images, for precise navigation of thoracoscopic sublobar lung resection. J Thorac Cardiovasc Surg 2014;147:1813-9.

8. Iwasaki Y, Nagata K, Yuba T, et al. Fluoroscopy-guided barium marking for localizing small pulmonary lesions before video-assisted thoracic surgery. Respir Med 2005;99:285-9.

9. Nomori H, Horio H, Naruke T, et al. Fluoroscopyassisted thoracoscopic resection of lung nodules marked with lipiodol. Ann Thorac Surg 2002;74:170-3.

10. Watanabe K, Nomori H, Ohtsuka T, et al. Usefulness and complications of computed tomography-guided lipiodol marking for fluoroscopy-assisted thoracoscopic resection of small pulmonary nodules: experience with 174 nodules. J Thorac Cardiovasc Surg 2006;132:320-4.

11. Ikeda K, Nomori H, Mori T, et al. Impalpable pulmonary nodules with ground-glass opacity: success for making pathologic sections with preoperative marking by lipiodol. Chest 2007:131:502-6.

12. Kawanaka K, Nomori H, Mori T, et al. Marking of small pulmonary nodules before thoracoscopic resection: injection of lipiodol under CT-fluoroscopic guidance. Acad Radiol 2009;16:39-45.

13. Kim YD, Jeong YJ, I H, et al. Localization of pulmonary nodules with lipiodol prior to thoracoscopic surgery. Acta Radiol 2011;52:64-9.

14. Miura H, Yamagami T, Tanaka O, et al. CT findings after lipiodol marking performed before video-assisted thoracoscopic surgery for small pulmonary nodules. Acta
Radiol 2016;57:303-10.

15. Mogi A, Yajima T, Tomizawa K, et al. Video-assisted thoracoscopic surgery after preoperative CT-guided lipiodol marking of small or impalpable pulmonary nodules. Ann Thorac Cardiovasc Surg 2015;21:435-9.

16. Mayo JR, Clifton JC, Powell TI, et al. Lung nodules: CT-guided placement of microcoils to direct videoassisted thoracoscopic surgical resection. Radiology 2009;250:576-85.

17. Park CH, Lee S, Lee J, et al. Hook-wire localization versus lipiodol localization for patients with pulmonary lesions having ground-glass opacity. J Thorac Cardiovasc Surg 2020;159:1571-9.e2.

18. Park CH, Han K, Hur J, et al. Comparative effectiveness and safety of pre-operative lung localization for pulmonary nodules: a systematic review and meta-analysis. Chest 2017;151:316-28.

19. Sawabata N, Ohta M, Matsumura A, et al. Optimal distance of malignant negative margin in excision of nonsmall cell lung cancer: a multicenter prospective study. Ann Thorac Surg 2004;77:415-20.

20. El-Sherif A, Fernando HC, Santos R, et al. Margin and local recurrence after sublobar resection of non-small cell lung cancer. Ann Surg Oncol 2007;14:2400-5.

21. Mohiuddin K, Haneuse S, Sofer T, et al. Relationship between margin distance and local recurrence among patients undergoing wedge resection for small $(\leq 2 \mathrm{~cm})$ non-small cell lung cancer. J Thorac Cardiovasc Surg 2014;147:1169-75.

22. Wolf AS, Swanson SJ, Yip R, et al. The impact of margins on outcomes after wedge resection for stage I non-small cell lung cancer. Ann Thorac Surg 2017;104:1171-8.

23. Nelson DB, Tayob N, Mitchell KG, et al. Surgical margins and risk of local recurrence after wedge resection of colorectal pulmonary metastases. J Thorac Cardiovasc Surg 2019;157:1648-55.

24. Masai K, Sakurai H, Sukeda A, et al. Prognostic impact of margin distance and tumor spread through air spaces in limited resection for primary lung cancer. J Thorac Oncol 2017;12:1788-97.

25. Fumimoto S, Sato K, Koyama M, et al. Combined lipiodol marking and video-assisted thoracoscopic surgery in a hybrid operating room. J Thorac Dis 2018;10:2940-7.

26. Hasegawa T, Kuroda H, Sato Y, et al. The utility of indigo carmine and lipiodol mixture for preoperative pulmonary nodule localization before video-assisted thoracic surgery. J Vasc Interv Radiol 2019;30:446-52.

27. Hsieh MJ, Fang HY, Lin CC, et al. Single-stage 
localization and removal of small lung nodules through image-guided video-assisted thoracoscopic surgery. Eur J Cardiothorac Surg 2018;53:353-8.

28. Zhao ZR, Lau RW, Ng CS. Hybrid theatre and alternative localization techniques in conventional and singleport video-assisted thoracoscopic surgery. J Thorac Dis 2016;8:S319-27.

Cite this article as: Fumimoto S, Sato K, Hanaoka N, Katsumata T. Identification of factors affecting the surgical margin in wedge resection using preoperative lipiodol marking. J Thorac Dis 2021;13(6):3383-3391. doi: 10.21037/jtd-21-211
29. Chao YK, Pan KT, Wen CT, et al. A comparison of efficacy and safety of preoperative versus intraoperative computed tomography-guided thoracoscopic lung resection. J Thorac Cardiovasc Surg 2018;156:1974-83.e1.

30. Sawabata N. Who should decide margin length in pulmonary excision of lung cancer? J Thorac Cardiovasc Surg 2014;148:370-1. 